\title{
Restricted Set Classification: Who is there?
}

\author{
Ludmila I. Kuncheva ${ }^{\mathrm{a}}$, Juan J. Rodríguez ${ }^{\mathrm{b}}$, Aaron S. Jackson ${ }^{\mathrm{c}}$ \\ ${ }^{a}$ Bangor University, Dean Street, Bangor Gwynedd, LL57 1UT, United Kingdom \\ ${ }^{b}$ University of Burgos, Escuela Politécnica Superior, Avda. de Cantabria s/n, 09006 Burgos, Spain \\ ${ }^{c}$ The University of Nottingham, NG8 1BB, United Kingdom
}

\begin{abstract}
We consider a problem where a set $X$ of $N$ objects (instances) coming from $c$ classes have to be classified simultaneously. A restriction is imposed on $X$ in that the maximum possible number of objects from each class is known, hence we dubbed the problem who-is-there? We compare three approaches to this problem: (1) Independent classification whereby each object is labelled in the class with the largest posterior probability; (2) A greedy approach which enforces the restriction; and (3) A theoretical approach which, in addition, maximises the likelihood of the label assignment, implemented through the Hungarian assignment algorithm. Our experimental study consists of two parts. The first part includes a custom-made chess data set where the pieces on the chess board must be recognised together from an image of the board. In the second part, we simulate the restricted set classification scenario using 96 datasets from a recently collated repository (University of Santiago de Compostela, USC). Our results show that the proposed approach (3) outperforms approaches (1) and (2).
\end{abstract}

Keywords: pattern recognition, object classification, restricted set classification, compound decision problem, chess pieces classification

\section{Introduction}

One of the standard assumptions in classical pattern recognition is that the data points to be classified come as an independent, identically distributed (iid) sequence. In many problems, this assumption does not hold. As an example, imagine the task of classifying all the pieces on a chess board from a bird-view napshot, without knowledge of the course of the game up to that position. A classifier trained to recognise each piece individually will not be aware that, say, there cannot be more than two white bishops on the board. Thus a white pawn could be misclassified as a white bishop without a penalty. Should the classifier 'know' the restriction, a mistake of this type will be less likely.

Email addresses: 1.i.kuncheva@bangor.ac.uk (Ludmila I. Kuncheva), jjrodriguez@ubu.es (Juan J. Rodríguez), aaron.jackson@nottingham.ac.uk (Aaron S. Jackson) 
Consider a classification problem where an instance $\mathbf{x}$ may come from one of the $c$ classes in the set $\Omega=\left\{\omega_{1}, \ldots, \omega_{c}\right\}$. Every instance is described by the values of $n$ features, so without loss of generality, $\mathbf{x} \in \mathbb{R}^{n}$. Let $X=\left\{\mathbf{x}_{1}, \ldots, \mathbf{x}_{c}\right\}$ be a set containing exactly one instance from each class. A set-classifier $D_{\text {set }}$ will label $X$ with a permutation of the $c$ class labels and ensure the best match in terms of classification accuracy [1, 2]. We refer to this task as "who-is-who".

This paper extends the above model to the more general case where $X$ consists of $m$ instances, and it is known that at most $k_{i}$ instances may belong to class $\omega_{i}, i=1, \ldots, c$. Denoting $k=k_{1}+\ldots+k_{c}$, we require that $m \leq k$. The who-is-who task is a special case where $k_{i}=1, i=1, \ldots, c$, and $m=c$.

Simultaneous classification of instances has been studied in various contexts for non-i.i.d data:

1. Compound decision problem. Duda et al. 3. formulate the problem where each class is represented in $X$ by a specific number of objects but without offering a solution. Taking inspiration from labelling the chromosomes in a cell (karyotyping), Slot 4 proposes a solution to this problem through maximising the log-likelihood of the labelling of $X$ by using 0-1 integer programming.

2. Multiple-instance classification. This problem arises in complex machine learning applications where the information about the instances is incomplete or ambiguous [5, 6, 7, 8, 9], for example, in drug activity prediction [5]. The training examples come in "bags" labelled either positive or negative. For a positive bag, it is known that at least one instance in the bag has a true positive label. For a bag labelled negative, all instances are known to be negative. The problem is to design a classifier that can label as accurately as possible an unseen bag of instances.

3. Set classification. In this problem, all the instances in a set are assumed to have come from the same unknown class [10. This problem may arise in face recognition where multiple images of the same person's 30 face are submitted as a set.

4. Relaxation labelling. The $m$ instances in set $X$ should be labelled using the label set $\Omega$. There are relationships between the classes which are specified by the so-called compatibility coefficients. Iterative relaxation labelling algorithms have been developed to solve this problem [11, 12,

Collective recognition. Collective recognition [13, 14] can be thought of as a special case of relaxation labelling. The instances within the set are related, so that the dependencies can be used to improve the classification accuracy. For example, in classifying web pages into topic categories, hyperlinked web pages are more likely to share common class labels than non-linked pages [14].

Who-is-who can be cast as a relaxation labelling problem. However, the compatibility coefficients enforcing the constraint of one-per-class are such that we cannot take advantage of the existing algorithms. In fact, there is an exact algorithm to solve who-is-who, which is preferable to the iterative alternatives. 
5. Tracking of multiple objects. Simultaneous classification of a set of instances is used in tracking algorithms for video sequences. For example, a moving object can be regarded as a patchwork of parts [15], a set of tracklets [16] or a structure with connected pieces such as parts of a human face or body [17, 18, 19, 20]. The parts are matched from one image frame to the next. Tracking several people in a video [17, 21, 22 also relies on simultaneous classification. The classification in tracking is dominated by assessing the spatial location of the object/part using algorithms such as Kalman filter, Probabilistic Data Association Filter (PDAF) 223, 24], mixture modelling, AdaBoost, particle filters [25, 26], temporal templates [17, the Hungarian algorithm [27, 28, a game-theory approach [22, a locomotion model 21] and so on.

The appearance-based component (which is the trained classifier in our model) is deemed much less important [19. Indeed, sometimes the objects are indistinguishable, and the only way to identify them is using their predicted and observed locations (for example, monitoring fruit flies 28]). Typically, the appearance-based classifier uses silhouette, texture [17, HSV colour histograms and edge detection [25, 23. Tracking piglets has been attempted by marking each piglet on the back by a dye pattern 29, 30, thereby empowering the appearance-based classifier. The simultaneous classification model proposed here can be regarded as an additional tool for improving the tracking accuracy by making a better use of the objects' appearance.

While close, none of the problems and solutions above matches exactly our formulation of the restricted set classification problem. The closest set-up is the compound decision problem but in our case we allow for up to $k_{i}$ objects from each class instead of a fixed number. Potential applications of the restricted set classification scenario include automatic attendance registration of students, karyotyping [31, 32, 33, 4, monitoring of animal behaviour (fruit flies [28, piglets [29, 30]), real-time labelling of the players in a game video stream (football [26], hockey [25]).

The rest of the paper is organised as follows. Section 2 lays out the theory behind the restricted set classification problem. Experimental results are shown in Section 3, and a conclusion is offered in Section 4

\section{The restricted set classification problem}

Definition 1. The restricted set classification problem is defined as follows. Let $X=\left\{\mathbf{x}_{1}, \ldots, \mathbf{x}_{m}\right\}$ be a set of instances such that at most $k_{i}$ instances come from class $\omega_{i} \in \Omega=\left\{\omega_{1}, \ldots, \omega_{c}\right\}$. Find labels for all elements of $X$ so that the restriction holds.

Note that $k_{1}+\ldots+k_{c}=k \geq m$.

Definition 2. A base classifier $D$ is a classifier that assigns a class label to an instance $\mathbf{x} \in \mathbb{R}^{n}$

$$
D: \mathbb{R}^{n} \rightarrow \Omega \text {. }
$$

We also require that $D$ provides estimates of the posterior probabilities $P\left(\omega_{1} \mid \mathbf{x}\right), \ldots, P\left(\omega_{c} \mid \mathbf{x}\right)$. 
Definition 3. A super-label for set $X$ is any collection of $m$ labels from $\Omega$ so that any instance $\mathbf{x} \in X$ receives a single label. A super-label will be called consistent if it satisfies the requirement that at most $k_{i}$ labels are equal to $\omega_{i}, i=1, \ldots, c$.

Denote by $\mathcal{S}$ the set of all possible super-labels of $X$. Let $P=\left[p_{i j}\right]$ be a matrix of size $m \times c$ that contains the posterior probability estimates obtained from the base classifier $D$ applied to $X$. Entry $p_{i j}$ is the estimate of $P\left(\omega_{j} \mid \mathbf{x}_{i}\right)$. Let $\mathcal{P}$ be the set of all matrices $P$.

Definition 4. A set classifier $D_{\text {set }}$ assigns a super-label to any set $X$ using the output of classifier $D$, that is

$$
D_{\text {set }}(X, D): \mathcal{P} \rightarrow \mathcal{S}
$$

We consider two type of estimates of the accuracy of $D_{\text {set }}$ for a given set $X$ :

- $A_{T}$, total accuracy: $A_{T}=1$ if all labels are correctly assigned to the instances in $X$, and $A_{T}=0$, otherwise;

- $A_{P}$, partial accuracy: $A_{P}$ is the proportion correctly labelled instances in $X$.

Definition 5. Independent (Baseline) Set Classifier $D_{\text {set }}^{i}$ This classifier takes the labels suggested by $D$ without any modification.

Definition 6. Greedy Set Classifier $D_{\text {set }}^{g}$. Assume that $D$ outputs the true posterior probabilities $P\left(\omega_{i} \mid \mathbf{x}\right)$, for $i=1 \ldots, c$ and any $\mathbf{x} \in \mathbb{R}^{n}$. This classifier labels the set $X$ according to the following algorithm:

1. Initialise a set $V=\emptyset$ to store the assigned object-class pairs.

2. Identify the largest posterior probability $P\left(\omega_{j}^{*} \mid \mathbf{x}_{j}^{*}\right)$ among the objects and classes not assigned so far.

3. Remove $\omega_{j}^{*}$ from the list of available classes, and $\mathbf{x}_{j}^{*}$ from the list of available objects, and add the pair to set $V$.

4. If there are no class-object pairs left, stop and return $V$. Else, continue from step 2.

To derive the extended model for simultaneous classification we will first introduce the two special cases: the who-is-who [1] and who-is-missing [2].

\subsection{Who-is-who?}

A set of $c$ objects have to be labelled into $c$ classes so that there is exactly one object in each class. Let $p$ be the probability that $D$ will label correctly a randomly chosen instance $\mathbf{x}$.

$D_{\text {set }}^{i}$ assumes that all instances are labelled independently. Then the accuracy measures of $D_{\text {set }}^{i}$ are

$$
A_{T}\left(D_{\text {set }}^{i}\right)=p^{c}
$$


and

$$
A_{P}\left(D_{\text {set }}^{i}\right)=p .
$$

The super-label assigned by $D_{\text {set }}^{i}$ may not be consistent, more likely so for larger number of classes $c$. It is possible to improve especially on $A_{P}\left(D_{\text {set }}^{i}\right)$ by ensuring that the super label is consistent, for example by applying $D_{\text {set }}^{g}$.

\subsubsection{Two classes}

Let $c=2$ and $X=\left\{\mathbf{x}_{1}, \mathbf{x}_{2}\right\}$. Without loss of generality, assume that $\mathbf{x}_{1}$ was drawn from the distribution of class $\omega_{1}$, and $\mathbf{x}_{2}$ from $\omega_{2}$, hence the true super-label is $\left\langle\omega_{1}, \omega_{2}\right\rangle$. Suppose again that $D$ is the perfect classifier for the chosen feature space, and therefore we have knowledge of the true posterior probabilities. To simplify notation, denote $P_{1}=P\left(\omega_{1} \mid \mathbf{x}_{1}\right)$ and $P_{2}=P\left(\omega_{2} \mid \mathbf{x}_{2}\right)$.

The probability that $D_{\text {set }}^{i}$ will give the correct super-label of $X$ is

$$
A_{T}\left(D_{\text {set }}^{i}(X)\right)=\operatorname{Pr}\left(P_{1}>0.5 \& P_{2}>0.5\right) .
$$

However, $D_{\text {set }}^{g}$ will add to this two more cases. The super-label will be correct also when $P_{1}<0.5$ and $P_{2}>1-P_{1}$, ensuring that $\omega_{2}$ will be assigned first to $\mathbf{x}_{2}$, leaving the free label $\omega_{1}$ for $\mathbf{x}_{1}$. By the same logic, $D_{\text {set }}^{g}$ will be right when $P_{2}<0.5$ and $P_{1}>1-P_{2}$. Since the cases are mutually exclusive, the probability that $D_{\text {set }}^{g}$ will give the correct super-label of $X$ is

$$
\begin{aligned}
A_{T}\left(D_{\text {set }}^{g}(X)\right) & = \\
& \operatorname{Pr}\left(P_{1}>0.5 \& P_{2}>0.5\right) \\
+ & \operatorname{Pr}\left(P_{1}<0.5 \& P_{2}>\left(1-P_{1}\right)\right) \\
+ & \operatorname{Pr}\left(P_{1}>\left(1-P_{2}\right) \& P_{2}<0.5\right) \\
\geq & A_{T}\left(D_{\text {set }}^{i}(X)\right)
\end{aligned}
$$

The above expression reduces to

$$
A_{T}\left(D_{\text {set }}^{g}(X)\right)=\operatorname{Pr}\left(P_{1}+P_{2}>0.5\right) .
$$

As $A_{T}\left(D_{\text {set }}^{g}(X)\right) \geq A_{T}\left(D_{\text {set }}^{i}(X)\right)$ for any $X$, the inequality is valid across the whole space of pairs $\left(\mathbf{x}_{1}, \mathbf{x}_{2}\right)$.

To visualise the improvement due to the greedy strategy, consider the two-dimensional data set shown in Figure 1. We drew 10,000 random pairs $X=\left\{\mathbf{x}_{1}, \mathbf{x}_{2}\right\} ; \mathbf{x}_{1}$ from $\omega_{1}$ and $\mathbf{x}_{2}$ from $\omega_{2}$.

The true probabilities $P_{1}=P\left(\omega_{1} \mid \mathbf{x}_{1}\right)$ and $P_{2}=P\left(\omega_{2} \mid \mathbf{x}_{2}\right)$ are used as the coordinate axes in Figure 2 where points corresponding to the 10,000 pairs are scattered. The region where $D_{\text {set }}^{i}$ gives the correct superlabel is shaded in light grey, and the number of points is shown. The regions where $D_{\text {set }}^{g}$ adds accuracy to that of $D_{\text {set }}^{i}$ are shaded in dark grey. 


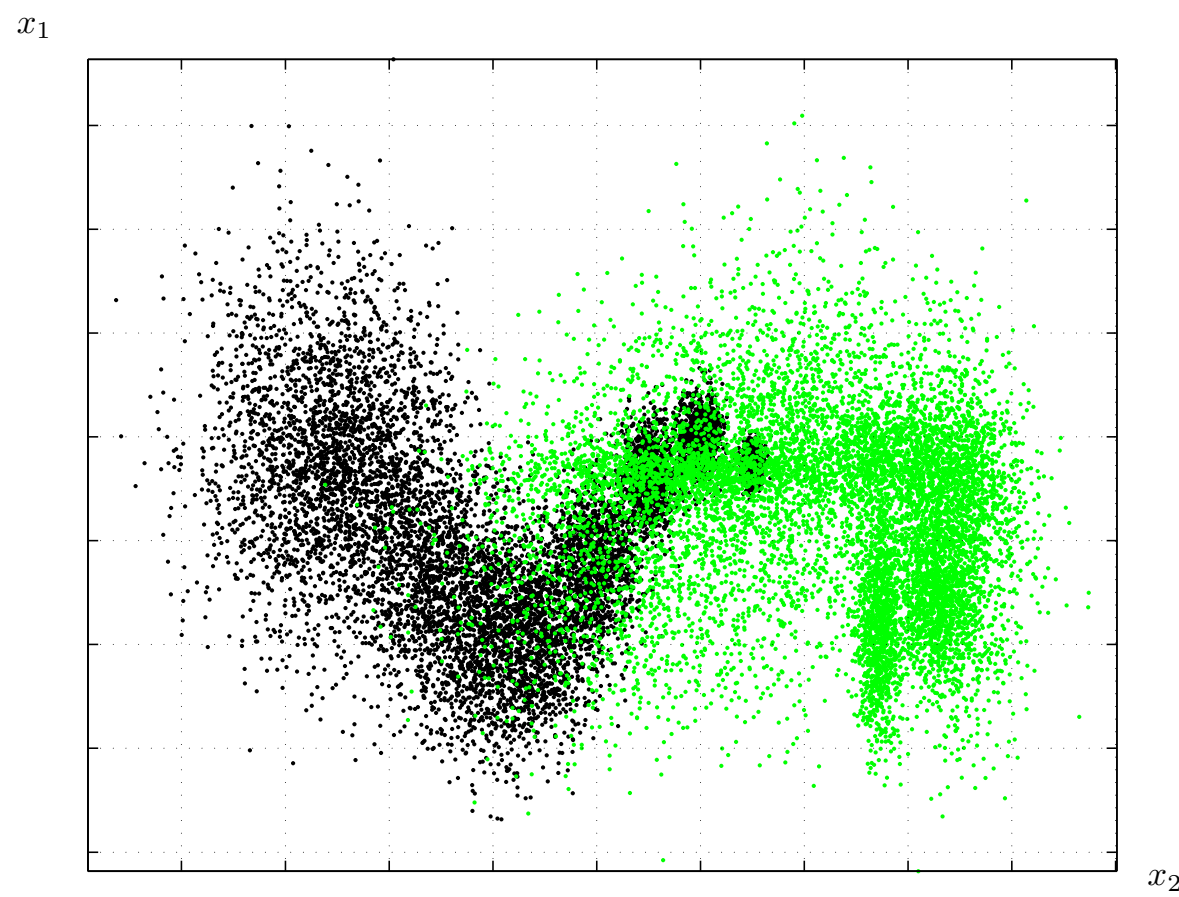

Figure 1: A two-dimensional data set with 10,000 points in each class.

For this example, $A_{T}\left(D_{\text {set }}^{i}\right)=80.80 \%$ and $A_{T}\left(D_{\text {set }}^{g}\right)=7.65+7.90+80.80=96.35 \%$.

Proposition 1. [1] For 2-class problems,

$$
A_{P}\left(D_{\text {set }}^{g}\right)>A_{P}\left(D_{\text {set }}^{i}\right)
$$

The proof is shown in the Appendix.

The theory about the two-class who-is-who problem has been illustrated by an earlier experimental study to demonstrate the results' validity when $D$ is not a Bayes classifier and the posterior probabilities are only estimates [1].

\subsection{2. $c$ classes}

Let $X=\left\{\mathbf{x}_{1}, \ldots, \mathbf{x}_{c}\right\}$ be the set of $c$ objects where object $\mathbf{x}_{i}$ is drawn from the distribution of class $\omega_{i}$, independently of the other $c-1$ objects. Then the likelihood of a super label $S=\left\langle s_{1}, \ldots, s_{c}\right\rangle, s_{i} \in \Omega$ is

$$
L(S)=\prod_{i=1}^{c} P\left(s_{i} \mid \mathbf{x}_{i}\right) p\left(\mathbf{x}_{i} \mid \omega_{i}\right)
$$

Since $p\left(\mathbf{x}_{i} \mid \omega_{i}\right)$ does not depend on the super-label $S$, we can organise it into a multiplicative constant $P_{X}=\prod_{i=1}^{c} p\left(\mathbf{x}_{i} \mid \omega_{i}\right)$, and rewrite the likelihood as

$$
L(S)=P_{X} \prod_{i=1}^{c} P\left(s_{i} \mid \mathbf{x}_{i}\right)
$$




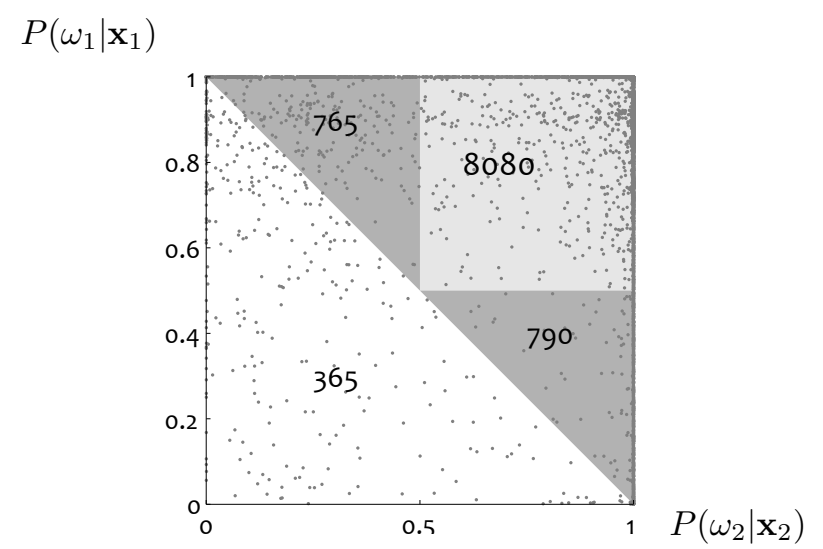

Figure 2: 10,000 random pairs of points with super-label $\left\langle\omega_{1}, \omega_{2}\right\rangle$ drawn from the problem in Figure 1 The points are plotted the space of the true posterior probabilities $P\left(\omega_{1} \mid \mathbf{x}_{1}\right)$ and $P\left(\omega_{2} \mid \mathbf{x}_{2}\right)$. The number of points in the respective regions are displayed.

The set of feasible super-labels is the set of all permutations of the elements of $\Omega$. The optimal super-label $S^{*}$ will be the one maximising the $L$ (equivalently $\log (L)$ ), that is

$$
S^{*}=\arg \max _{S \in \mathcal{I}} \sum_{i=1}^{c} \log \left(P\left(s_{i} \mid \mathbf{x}_{i}\right)\right),
$$

where $\mathcal{I}$ is the set of all permutations of the class labels in $\Omega$. $S^{*}$. Note that the greedy set classifier $D_{\text {set }}^{g}$ will not guarantee the optimal solution.

Definition 7. Hungarian Set Classifier $D_{\text {set }}^{h}$. This classifier uses the Hungarian assignment algorithm [34] to find $S^{*}$ The input to the algorithm is the matrix LP with the logarithms of the posterior probabilities obtained from the individual classifier, and the output is the optimal permutation $S^{*}$, guaranteeing the maximum sum of logarithms, as in equation 12 .

Previous experiments have shown significant improvement of $A_{T}\left(D_{\mathrm{set}}^{h}\right)$ over both $A_{T}\left(D_{\mathrm{set}}^{g}\right)$ and $A_{T}\left(D_{\mathrm{set}}^{i}\right)[1$.

\subsection{Who-is-Missing?}

In this scenario, a set $X$ of $k<c$ objects have to be labelled into $c$ classes so that there is at most one object in each class 22. As the question of interest here is "who-is-missing", it may be thought that the correct assignment of the elements of $X$ is not strictly necessary. However, in order to maximise the likelihood of discovering the identity of the missing classes, we still need to maximise the likelihood of the assignments of the objects in $X$. Let $S_{(-)} \subset \Omega$ and $S_{(+)}=\Omega \backslash S_{(-)}$be respectively the set of missing and the set of present classes. The probability that $S_{(-)}$is missing is the same as the probability that $S_{(+)}$is present.

\footnotetext{
${ }^{1}$ Further developed by Kuhn and Munkres, also known as Kuhn-Munkres algorithm. Proposed originally for $c \times c$ matrices, the Hungarian algorithm has been extended for rectangular matrices [35].
} 

of discovering the correct $S_{(-)}$. This allows for the who-is-missing problem to be cast as who-is-who.

We create $v=c-k$ dummy objects $\mathbf{z}_{1}, \ldots, \mathbf{z}_{v}$, and assign probabilities $P\left(\omega_{i} \mid \mathbf{z}_{j}\right)=1 / c$ for all $i=1, \ldots, c$ and $j=1, \ldots, v$. Thus the matrix with posterior probabilities $P$ is of size $c \times c$, and has $v$ identical rows with values $1 / c$. The optimal labelling (eqn 12 ) becomes

$$
S^{*}=\arg \max _{S \in \mathcal{I}}\left(\sum_{i=1}^{k} \log \left(P\left(s_{i} \mid \mathbf{x}_{i}\right)\right)+\sum_{j=1}^{v} \log \left(\frac{1}{c}\right)\right) .
$$
label. However, this time the set of classes has to be augmented with $k_{i}$ copies of each class. The posterior probabilities for the copies are the same as the one in the original column. In addition, the set of objects also has to be expanded to contain $k$ objects altogether. The $k-m$ dummy objects are assigned $1 / c$ posterior probabilities for all $k$ possible class labels in the super-label. The resultant matrix $P$ is of size $k \times k$. The example below illustrates this arrangement.

Consider three classes denoted respectively $\bullet, \triangle$ and $\square$. It is known that $X$ contains at most $k=5$ objects where at most 2 are from class $\bullet$, at most 1 is from class $\triangle$ and at most 2 are from class $\square$. Suppose that the observed set $X$ contains $m=4$ objects with the following posterior probabilities provided by $D$ : 


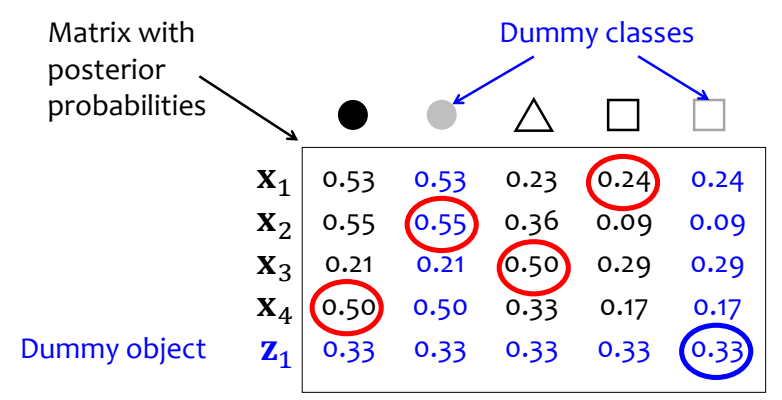

Figure 3: Construction of matrix $P(8 \times 8)$ for the numerical example. The ellipses show the assignment by $D_{\text {set }}^{h}$.

\begin{tabular}{c|ccc} 
Object & $P(\bullet \mid \mathbf{x})$ & $P(\triangle \mid \mathbf{x})$ & $P(\square \mid \mathbf{x})$ \\
\hline $\mathbf{x}_{1}$ & 0.53 & 0.23 & 0.24 \\
$\mathbf{x}_{2}$ & 0.55 & 0.36 & 0.09 \\
$\mathbf{x}_{3}$ & 0.21 & 0.50 & 0.29 \\
$\mathbf{x}_{4}$ & 0.50 & 0.33 & 0.17 \\
\hline
\end{tabular}

We construct $P$ as shown in Figure 3. The assignment resulting from applying $D_{\text {set }}^{h}$ to $P$ is also indicated in the figure.

The Hungarian set classifier $D_{\text {set }}^{h}$ will assign super-label $\langle\square \bullet \triangle \bullet\rangle$ to the four objects in $X$, amounting to criterion value

$$
\ln (0.24)+\ln (0.55)+\ln (0.50)+\ln (0.50)=-3.4112
$$

The Greedy set classifier $D_{\text {set }}^{g}$, on the other hand, will assign super-label $\langle\bullet \bullet \triangle \square\rangle$, which gives

$$
\ln (0.53)+\ln (0.55)+\ln (0.50)+\ln (0.17)=-3.6978
$$

Both super-labels satisfy the constraints but $D_{\text {set }}^{h}$ leads to a higher (better) log-likelihood value compared to $D_{\text {set }}^{g}$. This is to be expected as $D_{\text {set }}^{h}$ guarantees the optimal assignment of labels with respect to the log-likelihood criterion.

\section{Experiments}

Our hypothesis is that, for the restricted set classification problem, both accuracy measures $A_{T}$ and $A_{P}$ should be maximised by applying $D_{\text {set }}^{h}$ compared to applying $D_{\text {set }}^{g}$ and $D_{\text {set }}^{i}$, for various models of the base classifier $D$. 


\subsection{Chess pieces}

For this experiment we used 46 bird-view snapshots of a chess board. Each image was cropped and squared (Figure $4(\mathrm{a})$ ), and subsequently split into 64 squares. The task is to identify the chess pieces and for the chess pieces and one class for empty squares (denoted by E). Five instances (image tiles) are shown from each class.

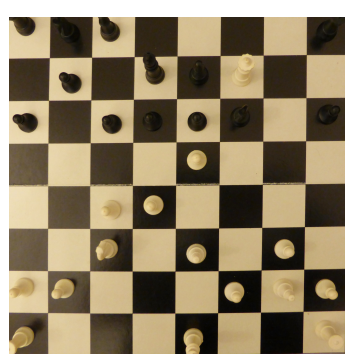

(a) Bird-view snapshot

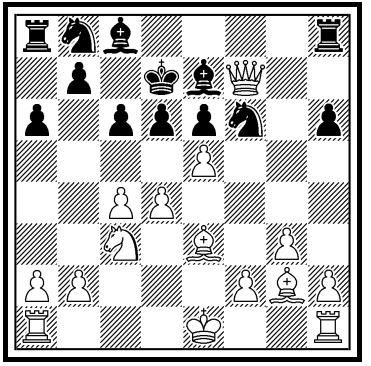

(b) True positions

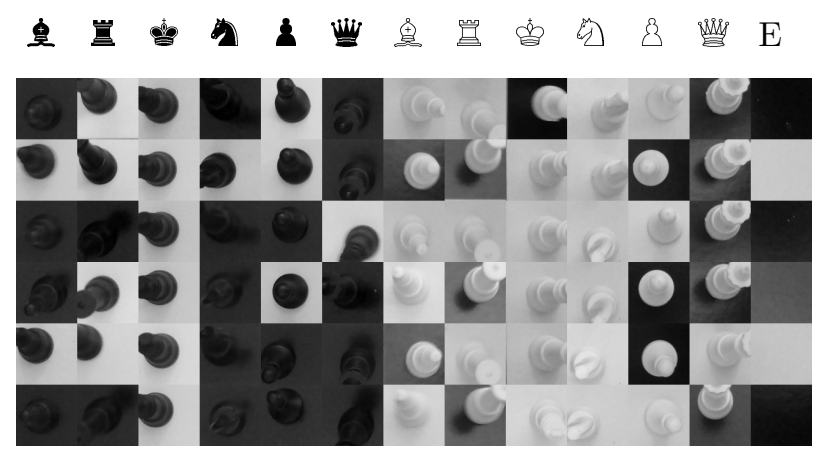

(c) Examples of the training data

Figure 4: A chess board example

We decided to use the following features on each tile:

- Entropy of the grey-level image.

- Standard deviation of the grey-level image.

- Difference between the mean grey intensity of (i) a square centred at the tile centre, with side equal to half of the tile side, and (ii) the tile area outside the square.

- 100 grey-level values obtained by resizing the image to $10 \times 10$ pixels. 
The experiment was organised as 46-fold cross-validation where one board was left aside in each fold. A classifier was trained for each fold, and the posterior probabilities of the testing data were subsequently calculated. The classifier models which we tried out as base classifier $D$ are shown in Table $1^{2}$

The matrix with the posterior probabilities $P$ was expanded as demonstrated in Figure 3 . The three set classifiers: $D_{\text {set }}^{i}(\mathrm{~B}), D_{\text {set }}^{g}(\mathrm{G})$ and $D_{\text {set }}^{h}(\mathrm{H})$ were then applied giving the accuracies shown in Table 1 .

Table 1: Averaged partial accuracy $A_{P}$ and averaged total accuracy $A_{T}$ in $\%$ for the Baseline (B), Greedy $(\mathrm{G})$ and the Hungarian $(\mathrm{H})$ set classifiers for the 46-cross-validation experiment for the Chess data. Symbol $\bullet$ after the value in column $A_{p}-\mathrm{H}$ indicates that $\mathrm{H}$ is significantly different from $\mathrm{G}$ at $p<0.001$, and symbol - means that there is no significant difference. The largest $A_{P}$ for each classifier type is underlined.

\begin{tabular}{r|rrr|rrr} 
& \multicolumn{3}{|c|}{ Partial accuracy $A_{P}$} & \multicolumn{3}{|c}{ Total accuracy $A_{T}$} \\
Classifier & $\mathrm{B}$ & $\mathrm{G}$ & $\mathrm{H}$ & $\mathrm{B}$ & $\mathrm{G}$ & $\mathrm{H}$ \\
\hline Nearest neighbour & 95.75 & 96.23 & $\underline{96.43}-$ & 32.61 & 43.48 & 47.83 \\
Linear discriminant analysis & 79.42 & 80.74 & $\underline{83.19} \bullet$ & 0.00 & 0.00 & 0.00 \\
Naïve Bayes & 39.64 & 59.68 & $\underline{64.20} \bullet$ & 0.00 & 0.00 & 0.00 \\
Decision tree & $\underline{87.13}$ & 85.67 & $\underline{86.01}-$ & 4.35 & 4.35 & 4.35 \\
SVM - linear & 89.57 & 90.18 & $\underline{90.52}-$ & 0.00 & 2.17 & 2.17 \\
SVM - radial basis function (RBF) & 87.67 & 88.52 & $\underline{89.50} \bullet$ & 2.17 & 2.17 & 2.17 \\
SVM - sigmoid kernel & 67.46 & $\underline{67.53}$ & $67.46-$ & 2.17 & 2.17 & 2.17 \\
SVM - polynomial kernel & 87.57 & 88.76 & $\underline{88.82}-$ & 0.00 & 0.00 & 0.00 \\
Random Forest & 91.88 & 93.00 & $\underline{93.58} \bullet$ & 2.17 & 19.57 & 26.09 \\
\hline
\end{tabular}

Paired t-test was used to identify any significant differences between $A_{P}$ for the proposed $D_{\text {set }}^{h}$ against $D_{\text {set }}^{g}$ at $p<0.001$ for the classifier models. The results favour $D_{\text {set }}^{h}$ which is often the most accurate set classifier. The greedy set classifier is the next best, and the baseline set classifier (independent application of $D$ ) wins only once, for the decision tree classifier. This is likely a result from the notoriously poor approximation of posterior probabilities by the standard decision tree classifier.

Looking at $A_{T}$, the only base classifier which can be of any use in recognising the whole set of chess pieces for this experiment is the nearest neighbour. Even its accuracy $A_{T}$ is not sufficiently high. For the purposes of demonstrating the advantage of using a proper set classifier, we chose a difficult task. This can be observed in the small example of the training set in Figure 4 (c). The position of the camera was such that the same type of piece could be seen to "lean" up, down, left or right, so much so that a distinguishing part of the top of the piece is missing. We did not attempt to analyse whether the background of the piece is

\footnotetext{
${ }^{2}$ Own MATLAB code was used for the nearest neighbour classifier, the naïve Bayes classifier and the random forest ensemble (RF). The MATLAB Statistic Toolbox was used for the linear discriminant classifier, the decision tree classifier and the random tree base classifier for RF. LibSVM library 36] was used for the multi-class SVM classifiers.
} 


\subsection{USC data}

\subsubsection{The data collection}

In the second set of experiments we used a collection of datasets chosen as a testbed for a comprehensive experimental evaluation of 179 classifiers from 17 families $37^{3}$. Many of the datasets within the collection are from the UCI Machine Learning Repository [38. We refer to this repository as USC after the host university (University of Santiago de Compostela, Spain).

To enable a reasonable formulation of the restricted set classification problem we had to ensure that there is sufficient variability within each class we sampled from. Otherwise the results would depend too much on a few instances. Therefore, we reduced the original 121 datasets to 96 datasets, and modified those retained according to the following rule. Classes containing less than 50 examples were removed. Hence, datasets without at least two classes with at least 50 examples in each were discarded from the collection. The description of the data collection that we used is shown in Table 2 .

\subsubsection{Experimental protocol}

Two fold cross validation, repeated five times, was used to partition the data into training and initial testing sets. From each of the ten initial testing sets, 100 runs were carried out. Thus, for each dataset, we carried out 1000 runs.

In each run, we commence by initialising the set to be labelled by $X=\emptyset$. A random integer between 1 and 10 is drawn for each class to serve as the limit on the number of possible objects from that class. Denote by $k_{i}$ the number of objects allowed for class $\omega_{i}$. Then a second random integer, $r_{i}$, is drawn between 0 and $k_{i}$, to determine how many objects from $\omega_{i}$ will actually be present in the set $X$. Note that we include the possibility of a completely absent class. Next, we add to $X r_{i}$ randomly selected testing objects whose true label is $\omega_{i}$. After constructing the set $X, D_{\text {set }}^{i}$ (Baseline), $D_{\text {set }}^{g}$ (Greedy) and $D_{\text {set }}^{h}$ (Hungarian) are applied to find the respective super-labels.

The following set of classifiers were tested as $D$ :

- Nearest neighbour (1-NN)

\footnotetext{
${ }^{3}$ The repository is available at http://persoal.citius.usc.es/manuel.fernandez.delgado/papers/jmlr/
} 
Table 2: Characteristics of the datasets (\#E: examples, \#F: feautures, \#C: classes).

\begin{tabular}{|c|c|c|c|c|c|c|c|}
\hline dataset & $\# \mathrm{E}$ & $\# \mathrm{~F}$ & $\# \mathrm{C}$ & dataset & $\# \mathrm{E}$ & $\# \mathrm{~F}$ & $\# \mathrm{C}$ \\
\hline abalone & 4177 & 8 & 3 & molec-biol-splice & 3190 & 60 & 3 \\
\hline acute-inflammation & 120 & 6 & 2 & monks-1 & 556 & 6 & 2 \\
\hline acute-nephritis & 120 & 6 & 2 & monks-2 & 601 & 6 & 2 \\
\hline adult & 48842 & 14 & 2 & monks-3 & 554 & 6 & 2 \\
\hline annealing & 850 & 31 & 3 & mushroom & 8124 & 21 & 2 \\
\hline arrhythmia & 295 & 262 & 2 & musk-1 & 476 & 166 & 2 \\
\hline balance-scale & 576 & 4 & 2 & musk-2 & 6598 & 166 & 2 \\
\hline bank & 4521 & 16 & 2 & nursery & 12958 & 8 & 4 \\
\hline blood & 748 & 4 & 2 & oocytes-merluccius-nucleus- $4 \mathrm{~d}$ & 1022 & 41 & 2 \\
\hline breast-cancer & 286 & 9 & 2 & oocytes-merluccius-states- $2 \mathrm{f}$ & 1022 & 25 & 3 \\
\hline breast-cancer-wisc & 699 & 9 & 2 & oocytes-trisopterus-nucleus- $2 \mathrm{f}$ & 912 & 25 & 2 \\
\hline breast-cancer-wisc-diag & 569 & 30 & 2 & oocytes-trisopterus-states-5b & 898 & 32 & 2 \\
\hline car & 1728 & 6 & 4 & optical & 5620 & 62 & 10 \\
\hline cardiotocography-10clases & 2126 & 21 & 10 & ozone & 2536 & 72 & 2 \\
\hline cardiotocography-3clases & 2126 & 21 & 3 & page-blocks & 5445 & 10 & 4 \\
\hline chess-krvk & 28029 & 6 & 17 & pendigits & 10992 & 16 & 10 \\
\hline chess-krvkp & 3196 & 36 & 2 & pima & 768 & 8 & 2 \\
\hline congressional-voting & 435 & 16 & 2 & planning & 182 & 12 & 2 \\
\hline conn-bench-sonar-mines-rocks & 208 & 60 & 2 & ringnorm & 7400 & 20 & 2 \\
\hline conn-bench-vowel-deterding & 990 & 11 & 11 & seeds & 210 & 7 & 3 \\
\hline connect-4 & 67557 & 42 & 2 & semeion & 1593 & 256 & 10 \\
\hline contrac & 1473 & 9 & 3 & soybean & 362 & 35 & 4 \\
\hline credit-approval & 690 & 15 & 2 & spambase & 4601 & 57 & 2 \\
\hline cylinder-bands & 512 & 35 & 2 & spect & 265 & 22 & 2 \\
\hline dermatology & 297 & 34 & 4 & spectf & 267 & 44 & 2 \\
\hline ecoli & 272 & 7 & 3 & statlog-australian-credit & 690 & 14 & 2 \\
\hline energy-y1 & 768 & 8 & 3 & statlog-german-credit & 1000 & 24 & 2 \\
\hline energy-y2 & 768 & 8 & 3 & statlog-heart & 270 & 13 & 2 \\
\hline glass & 146 & 9 & 2 & statlog-image & 2310 & 18 & 7 \\
\hline haberman-survival & 306 & 3 & 2 & statlog-landsat & 6435 & 36 & 6 \\
\hline hayes-roth & 129 & 3 & 2 & statlog-shuttle & 57977 & 9 & 5 \\
\hline heart-cleveland & 219 & 13 & 2 & statlog-vehicle & 846 & 18 & 4 \\
\hline heart-hungarian & 294 & 12 & 2 & steel-plates & 1941 & 27 & 7 \\
\hline heart-va & 107 & 12 & 2 & synthetic-control & 600 & 60 & 6 \\
\hline hill-valley & 1212 & 100 & 2 & teaching & 102 & 5 & 2 \\
\hline horse-colic & 368 & 25 & 2 & thyroid & 7200 & 21 & 3 \\
\hline ilpd-indian-liver & 583 & 9 & 2 & tic-tac-toe & 958 & 9 & 2 \\
\hline image-segmentation & 2310 & 18 & 7 & titanic & 2201 & 3 & 2 \\
\hline ionosphere & 351 & 33 & 2 & twonorm & 7400 & 20 & 2 \\
\hline iris & 150 & 4 & 3 & vertebral-column-2clases & 310 & 6 & 2 \\
\hline led-display & 1000 & 7 & 10 & vertebral-column-3clases & 310 & 6 & 3 \\
\hline letter & 20000 & 16 & 26 & wall-following & 5456 & 24 & 4 \\
\hline low-res-spect & 469 & 100 & 3 & waveform & 5000 & 21 & 3 \\
\hline lymphography & 142 & 18 & 2 & waveform-noise & 5000 & 40 & 3 \\
\hline magic & 19020 & 10 & 2 & wine & 130 & 13 & 2 \\
\hline mammographic & 961 & 5 & 2 & wine-quality-red & 1571 & 11 & 4 \\
\hline miniboone & 130064 & 50 & 2 & wine-quality-white & 4873 & 11 & 5 \\
\hline molec-biol-promoter & 106 & 57 & 2 & yeast & 1350 & 8 & 5 \\
\hline
\end{tabular}


- Linear discriminant analysis (LDA)

- Naïve Bayes (NB)

- Logistic Regression (LOG)

- Decision tree (DT)

- Random Forest (RF)

- Rotation Forest (ROT)

\subsubsection{Results}

We have prepared a supplementary document which contains the full numerical results from the experiments in seven tables, one for each classifier. Here we reproduce only the table for the decision tree classifier (Table 4).

The best and the worst base classifiers. We next give a graphical illustration of the two methods which appeared to be the weakest and the strongest in our experiment: Naïve Bayes (NB, the weakest) and Rotation Forest ensemble (ROT, the strongest). Figure 5 (a) shows the improvement on $A_{P}$ of $D_{\text {set }}^{i}$ achieved by applying $D_{\text {set }}^{h}$ to the restricted set classification problem. To prepare the plot, we arranged the datasets in ascending order of $A_{P}\left(D_{\text {set }}^{i}\right)$. Then we plotted $A_{P}\left(D_{\text {set }}^{i}\right)$ and $A_{P}\left(D_{\text {set }}^{h}\right)$ versus the dataset index. The differences between the two curves are small and not clearly visible, especially for larger values of $A_{P}\left(D_{\text {set }}^{i}\right)$. Therefore, in order to show the consistency of the improvement, we drew a vertical line upwards from each point (dataset) where the strict inequality $A_{P}\left(D_{\text {set }}^{i}\right)<A_{P}\left(D_{\text {set }}^{h}\right)$ held. Shown above the curves is the number of datasets out of 96 which satisfy the inequality. For NB, the partial accuracy $A_{P}\left(D_{\text {set }}^{h}\right)$ was higher than $A_{P}\left(D_{\text {set }}^{i}\right)$ for all 96 datasets.

In the same way, we plot together the curves for $A_{P}\left(D_{\text {set }}^{g}\right)$ and $A_{P}\left(D_{\text {set }}^{h}\right)$, this time sorting the datasets on $A_{P}\left(D_{\text {set }}^{g}\right)$. The graph is shown in Figure 5 (b). This time, there were datasets for which the opposite strict inequality held, that is, $A_{P}\left(D_{\text {set }}^{g}\right)>A_{P}\left(D_{\text {set }}^{h}\right)$. For these datasets, we drew the vertical lines downward, and show the number of datasets under the curves. For this case, Method $H$ was better than method $G$ in 42 comparisons and worse in 13 comparisons, leaving 41 ties. We can apply the sign statistical test whose p-value signifies reflects the probability that such difference may happen by chance if the two methods are, in fact, equivalent. For the results plotted in Figure 5 (b), $p=6.66 \times 10^{-5}$, suggesting that $A_{P}\left(D_{\text {set }}^{h}\right)$ is significantly better than $A_{P}\left(D_{\text {set }}^{g}\right)$.

Figure 5 suggests that, even though $A_{P}$ is not as high as for the remaining baseline classifiers $D$, the set classifier is able to improve on it consistently. Moreover, the Hungarian algorithm should be preferred to the greedy algorithm for its, albeit small, provably superior accuracy. 


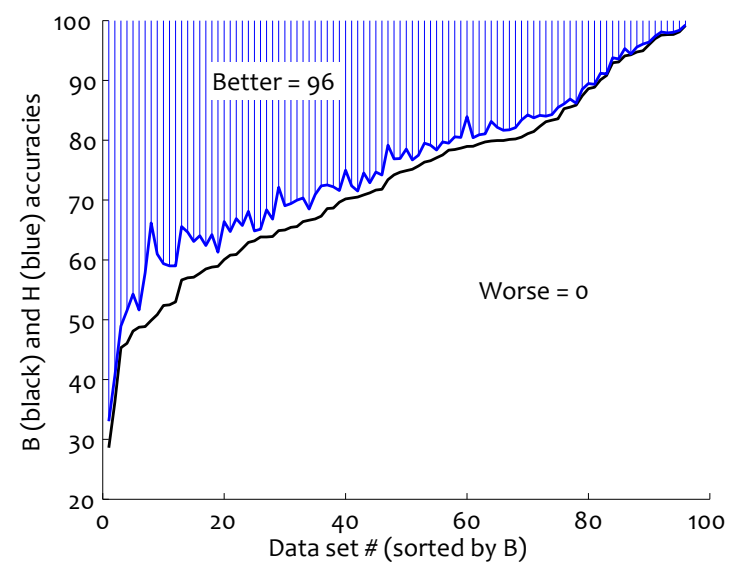

(a)

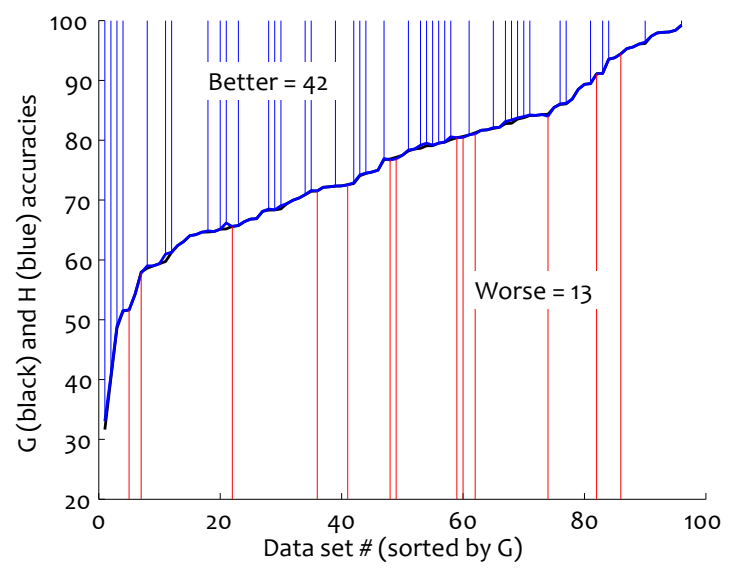

(b)

Figure 5: Comparison between the partial accuracy $A_{P}$ of: (a) $D_{\text {set }}^{i}$ and $D_{\text {set}}^{h}$, and (b) $D_{\text {set }}^{g}$ and $D_{\text {set }}^{h}$ for the Naïve Bayes classifier and USC data collection. Upward vertical lines indicate that method $H$ is strictly better than its counterpart for the respective dataset, and downward vertical line indicate that it is strictly worse. The numbers of datasets which satisfy the inequalities are shown in the respective parts of the plot.

Figure 6 is a matching example to Figure 5 where the based classifier is the Rotation Forest ensemble, the best overall set classifier. Method $H$ is again making a "clean sweep" against $I$. This time, however, the number of results where method $H$ is worse than method $G$ rose to 38 versus 55 comparisons in favour of method $H$. This gives $p=0.0180$ for the two-tailed sign test, which still supports the claim that there is significant difference in favour of method $H$ at $p<0.01$.

The scaling of the figures was kept the same to allow for a visual comparison of the accuracies. Clearly, ROT leads to better $A_{P}$ than NB as all curves run higher in Figure 6. The improvement on $A_{P}$, however, is more pronounced for NB. Part of this may be due to the fact that NB is meant to approximate posterior probabilities (under the feature independence assumption), and gives a ready-made matrix with probabilities $P$. Rotation Forest, on the other hand, uses the voting scores as approximation of the posterior probabilities, which is not ideal, and may compromise the expected improvement on the accuracy of the independent set classifier $I$,

Figure 7 contains all the results for the total accuracy $A_{T}$ whereby we require that all elements of $X$ are classified correctly (correct super-label). The p-values for the results in subplots (b) and (d) are respectively 0.0820 and 0.4484 . If we consider the right-tailed test with null hypothesis about the medians $G \geq H$, and alternative $H>G$, then the p-value for subplot (b) is 0.0410 , hence method $H$ is better than method $G$ at $p<0.05$.

Statistical testing for all results. Table 3 presents a summary of all the results. Given the large number of datasets, we considered it reasonable to display the average accuracies to support the statistical test 

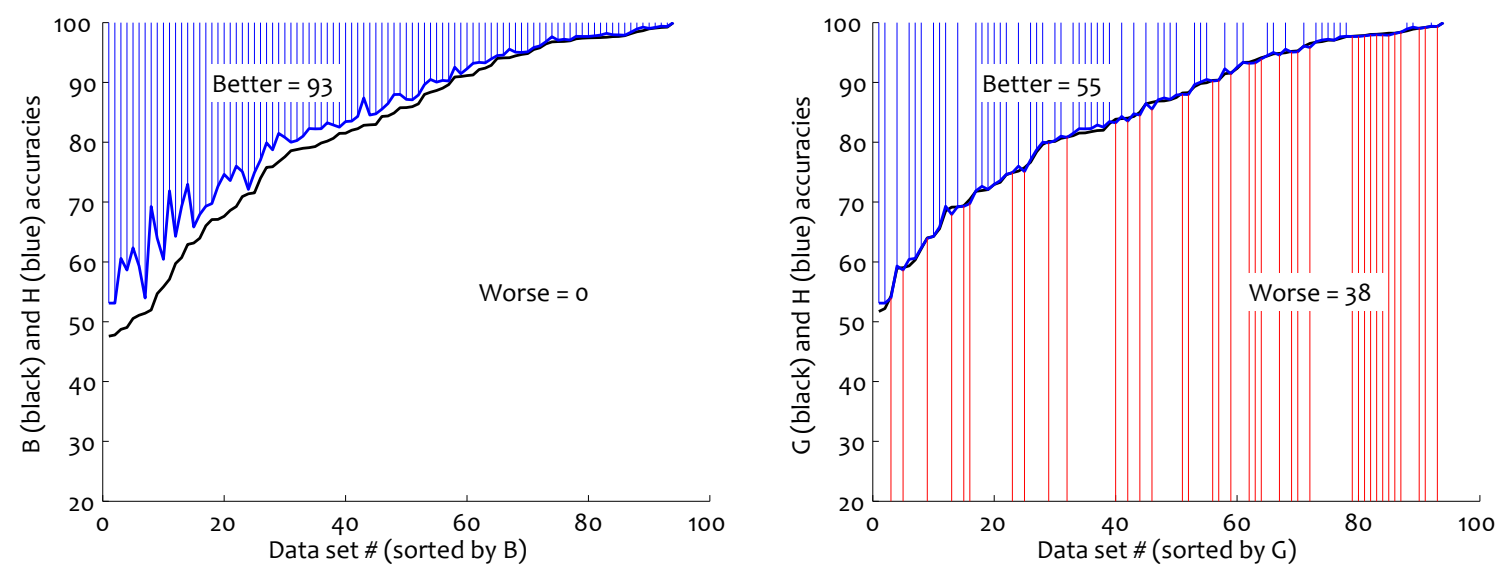

Figure 6: Comparison between the partial accuracy $A_{P}$ of: (a) $D_{\text {set }}^{i}$ and $D_{\text {set }}^{h}$, and (b) $D_{\text {set }}^{g}$ and $D_{\text {set }}^{h}$ for the Rotation Forest ensemble and USC data collection. Upward vertical lines indicate that method $H$ is strictly better than its counterpart for the respective dataset, and downward vertical line indicate that it is strictly worse. The numbers of datasets which satisfy the inequalities are shown in the respective parts of the plot.

results. Note that we still used the non-parametric sign test for the comparison. The results support the claim that the set classifier which uses the Hungarian assignment algorithm is consistently better than the two rival algorithms. Admittedly, while consistent, the improvement over method $G$ is fairly small, as evidenced by Figures 5-7, where the curves for $G$ and $H$ visibly coincide. This raises up the question of whether the Hungarian algorithm is really needed for this task or satisfactory results can be obtained with the greedy algorithm?

Note that this paper is about the definition of the restricted set classification problem. The crucial part of the proposed solution is the expansion of the probability matrix $P$. This can be followed by the optimal assignment algorithm $(H)$ or a good suboptimal assignment algorithm $(G)$.

Analysis of the improvement of $H$ over $B$. Figure 8 illustrates the improvement offered by method $H$ over the baseline set classifier $B$ for both partial accuracy $A_{P}$ and total accuracy $A_{T}$. For this example we used all the results for the seven base classifiers. Each point corresponds to a dataset, so there are $96 \times 7=672$ points scattered in total in each sub-plot. The diagonal line is where the points should lie if $H$ and $B$ had identical accuracies. The figure shows that, for both $A_{P}$ and $A_{T}, H$ is better than $B$. Interestingly, the improvement on $A_{P}$ (sub-plot (a)) depends on the base accuracy while the improvement on $A_{T}$ (sub-plot (b)) is more uniform on the total accuracy of $B$. The set classifier can correct individual errors better when the base classifier is not very accurate, and less so when the individual accuracy increases. This tendency exists but is less pronounced for the total accuracy $A_{T}$.

In Figure 9 we examine the relationship between the improvement on the partial and the total accuracy. The 672 points obtained from all seven classifier models and all datasets are scattered in the space 


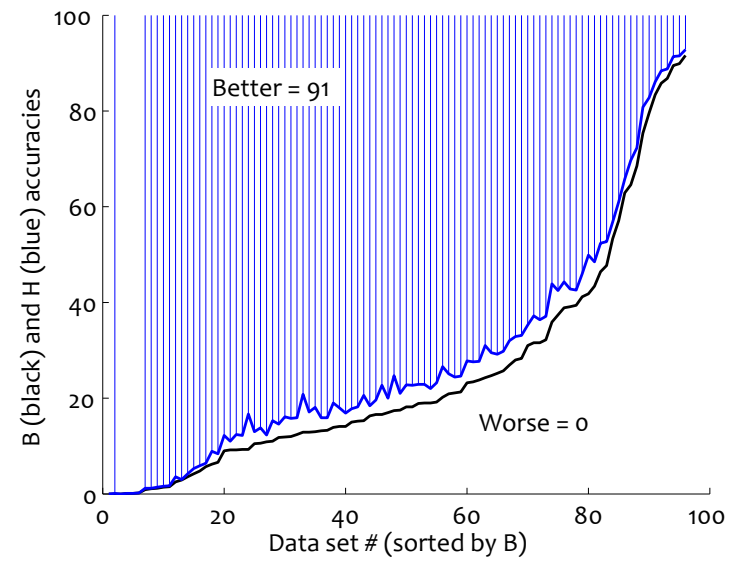

(a)

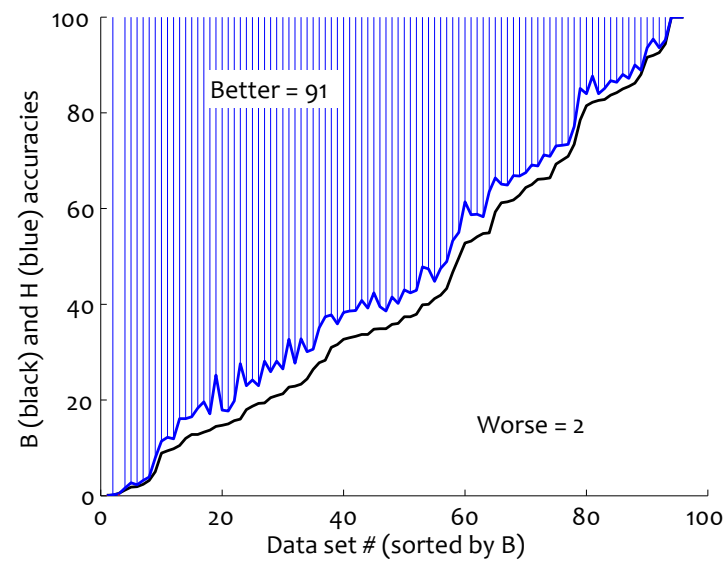

(c)

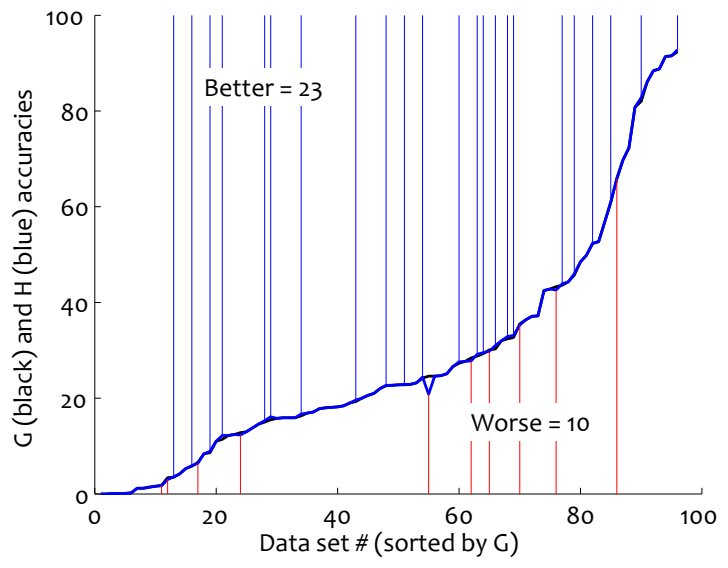

(b)

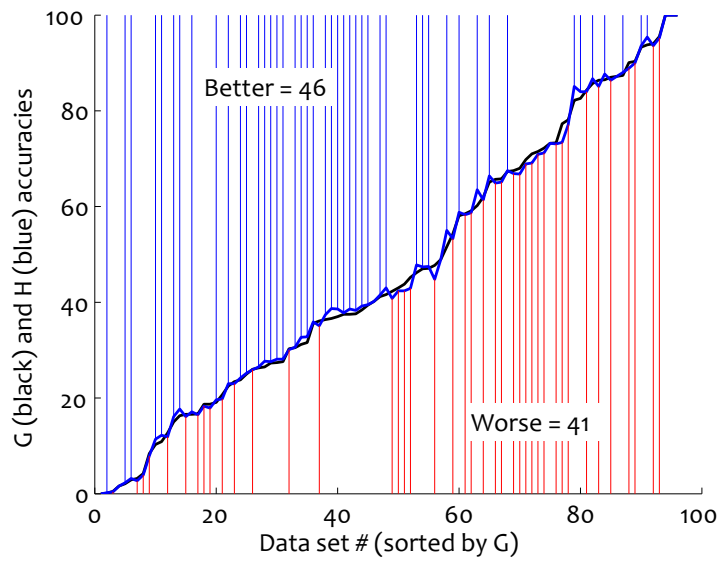

(d)

Figure 7: Comparison between the total accuracy $A_{T}$ of: (a) method $H$ versus $I$, (b) method $H$ versus method $G$ for the Naïve Bayes classifier; (c) method $H$ versus $I$, (d) method $H$ versus method $G$ for the Rotation Forest ensemble, all for the USC data collection. Upward vertical lines indicate that method $H$ is strictly better than its counterpart for the respective dataset, and downward vertical line indicate that it is strictly worse. The numbers of datasets which satisfy the inequalities are shown in the respective parts of the plot. 
Table 3: Average partial accuracy $A_{P}$ and total accuracy $A_{T}$ for the three set classifiers ( $B$ Baseline, $G$ Greedy and $H$ Hungarian) for seven base classifier models with the USC data collection. Method $H$ was compared with $I$ and $G$ using the sign test at significance level $p<0.001$. The results are shown next to the $H$ columns. The first symbol is the result of the $H / I$ comparison, and the second symbol, the result from the $H / G$ comparison. A bullet indicates that $H$ is significantly better that the other set classifier, and a dash indicates that there is no difference at the chosen significance level.

\begin{tabular}{r|rrr|rrr} 
& \multicolumn{3}{|c|}{ Partial accuracy $A_{P}$} & \multicolumn{3}{|c}{ Total accuracy $A_{T}$} \\
Classifier & B & G & H & B & G & H \\
\hline Nearest neighbour (1-NN) & 76.05 & 77.57 & $78.14 \bullet \bullet$ & 30.03 & 32.55 & $33.45 \bullet \bullet$ \\
Linear discriminant analysis (LDA) & 73.74 & 76.86 & $76.99 \bullet \bullet$ & 27.67 & 30.97 & $31.06 \bullet \bullet$ \\
Naïve Bayes (NB) & 72.83 & 75.86 & $75.95 \bullet \bullet$ & 25.10 & 28.50 & $28.51 \bullet-$ \\
Logistic Regression (LOG) & 75.13 & 78.43 & $78.74 \bullet \bullet$ & 29.74 & 33.65 & $33.95 \bullet \bullet$ \\
Decision tree (DT) & 77.81 & 79.85 & $80.27 \bullet \bullet$ & 32.63 & 35.60 & $36.29 \bullet \bullet$ \\
Random Forest (RF) & 81.25 & 83.57 & $83.62 \bullet \bullet$ & 40.16 & 44.03 & $44.14 \bullet \bullet$ \\
\hline
\end{tabular}

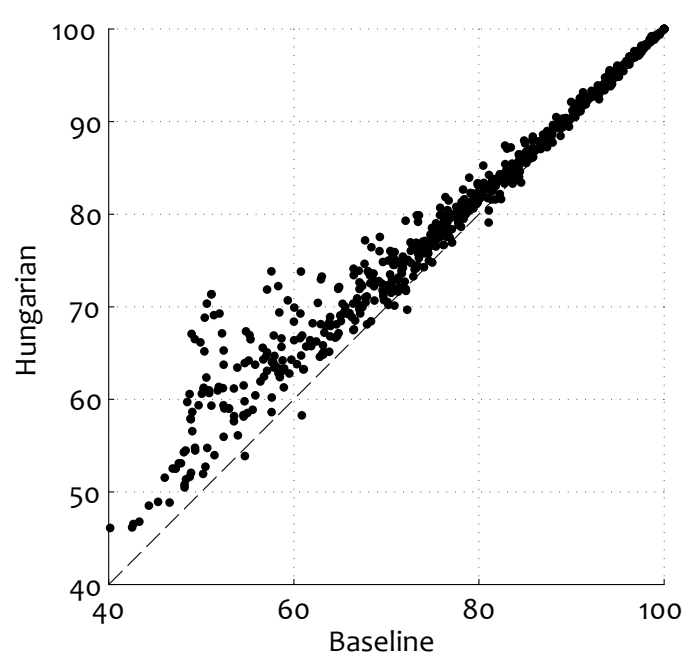

(a) Partial accuracy $A_{P}$

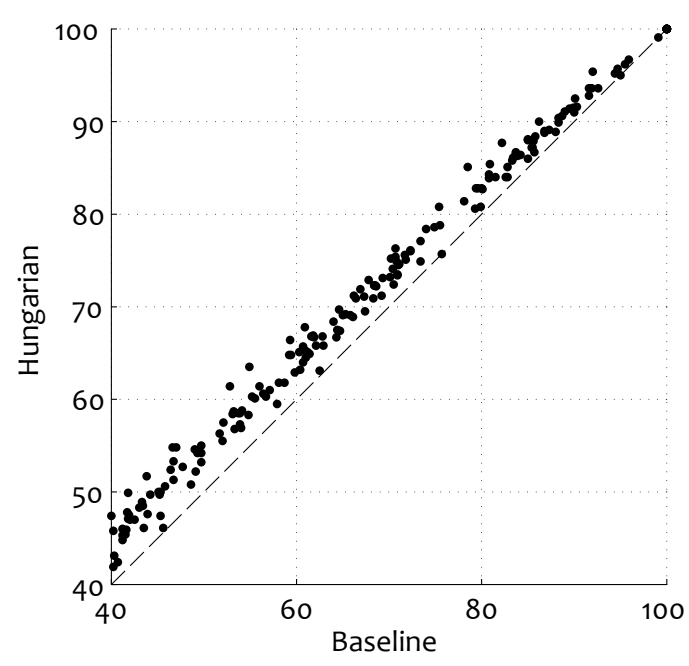

(b) Total accuracy $A_{T}$

Figure 8: Improvement of method $H$ over method $B$ for the 96 USC datasets using the all 7 base classifiers. 


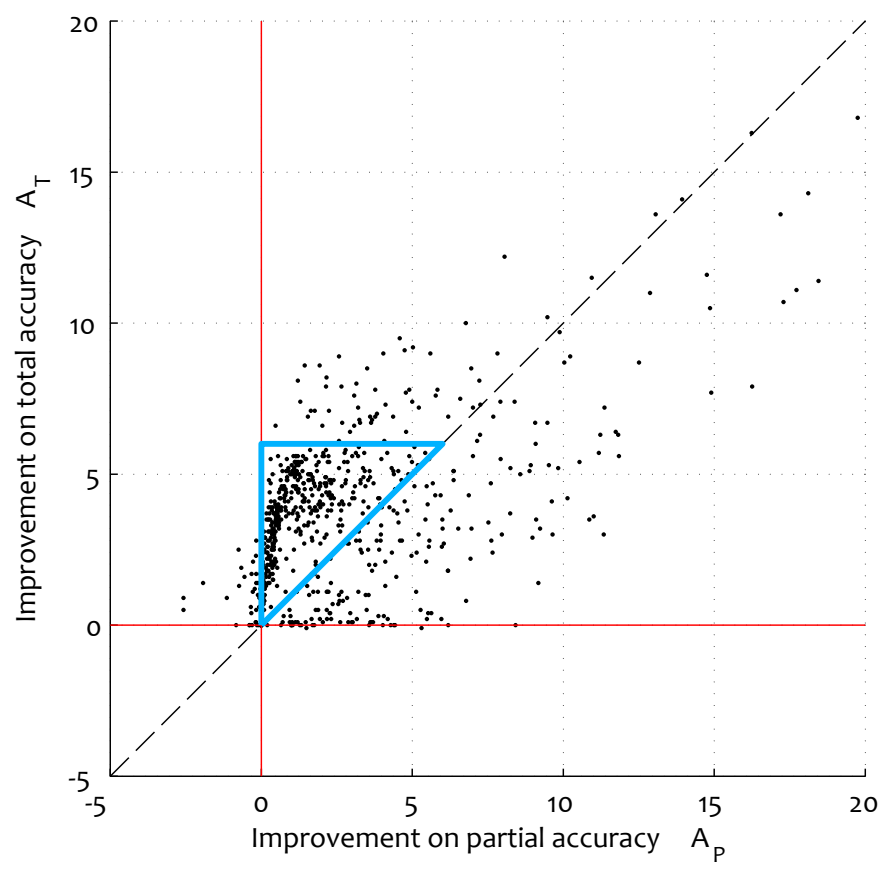

Figure 9: Scatterplot of all datasets for all seven base classifiers in the plane $\left(\Delta A_{P}, \Delta A_{T}\right)$. The blue triangle contains $54 \%$ of all points

$\left(\Delta A_{P}, \Delta A_{T}\right)$ where $\Delta A_{P}=A_{P}\left(D_{\text {set }}^{h}\right)-A_{P}\left(D_{\text {set }}^{i}\right)$ and $\Delta A_{T}=A_{T}\left(D_{\text {set }}^{h}\right)-A_{T}\left(D_{\text {set }}^{i}\right)$. The lines of no improvement are depicted in red. The figure shows that the total accuracy benefits even more than the partial accuracy $\left(68.6 \%\right.$ of the points are above the diagonal line, that is $\left.\Delta A_{T}>\Delta A_{P}\right)$. We identified and outlined by a triangle a dense region in the diagram containing approximately $54 \%$ of all points plotted. For these points, a fairly small improvement on the partial accuracy was sufficient to ensure a greater improvement in the total accuracy, justifying overall the restricted set classification approach. Larger improvements on $A_{P}$ lead to larger improvement on $A_{T}$ too, but for most of these points, $\Delta A_{P}>\Delta A_{T}$ (that is, the points lie under the diagonal).

Another interesting observation from the figure is that even for datasets where the partial accuracy of method $H$ was worse than that of method method $B$ (left from the line $\Delta A_{P}=0$ ), the total accuracy of method $H$ was still better than that of method $B\left(\Delta A_{T}>0\right)$. Overall, $\Delta A_{T}>0$ for $93.75 \%$ of the points, $\Delta A_{T}=0$ for $5.95 \%$ of the points, and $\Delta A_{T}<0$ for $0.3 \%$ of the points. Note that this set of points includes all seven base classifiers and all datasets.

Relationship of the improvement of $H$ over $B$ and the number of classes.. Figure 10 plots the improvement $\Delta A_{P}$ and $\Delta A_{T}$ versus the number of classes. The points in the scatterplots again correspond to the datasets using all seven base classifiers.

There is no visible relationship between the number of classes and the improvement of method $H$ against 


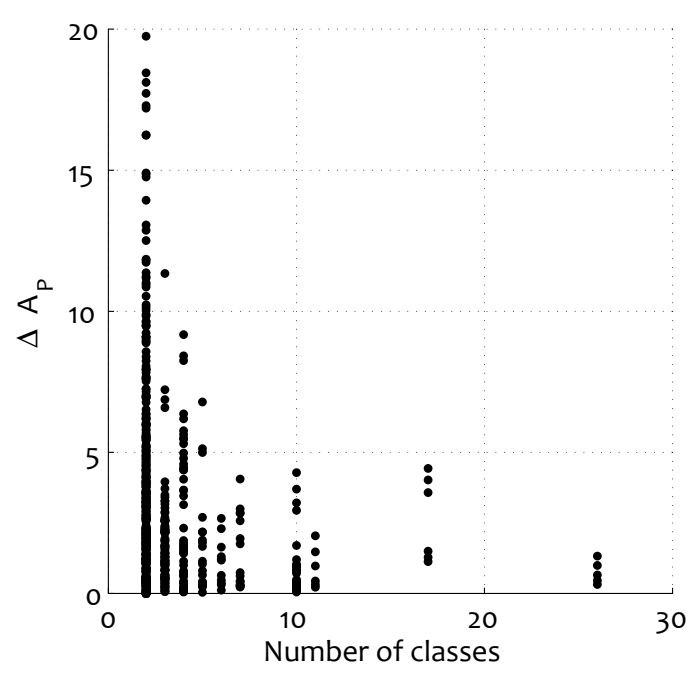

(a)

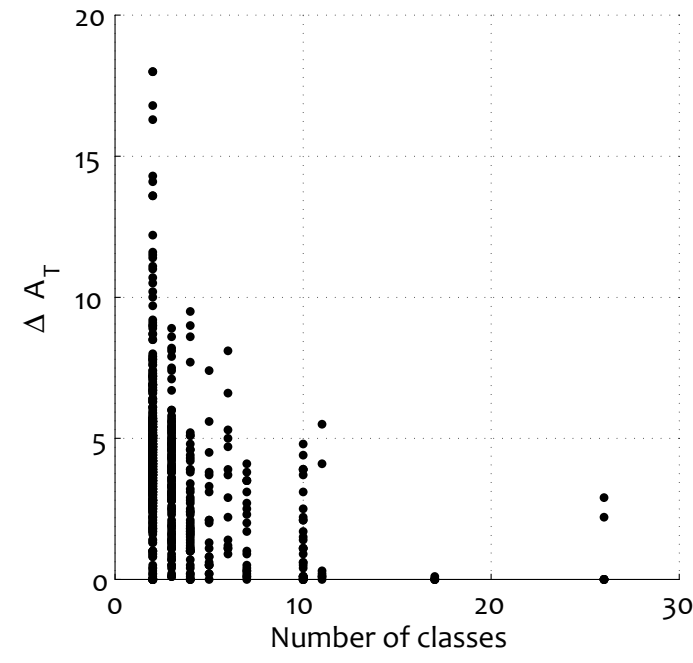

(b)

Figure 10: Improvement of $H$ over $B$ for the partial accuracy $\left(\Delta A_{P}\right)$ and the total accuracy $\left.\Delta A_{T}\right)$ against the number of classes for the respective problems.

method $B$. The problems with two and three classes make up $75 \%$ of the USC collection. It can be expected that more is to be gained if we have a large number of classes compared to fewer classes, but this could not be verified with this collection.

\section{Conclusion}

We propose a solution for the restricted set classification problem which we also call "who-is-there". The problem is to classify simultaneously a set of objects into $c$ classes, $\omega_{1}, \ldots, \omega_{c}$, knowing that there is at most $k_{i}$ objects from class $\omega_{i}$. The values of $k_{i}$ are specified in advance and fixed. Our solution is to expand the matrix with posterior probabilities given by the classifier so that we cover the possibility for $k=\sum_{i=1}^{c} k_{i}$ objects and labels, and apply the Hungarian assignment algorithm on the logarithms of the posterior probabilities in the expanded matrix. The simpler alternatives which we considered were the standard approach of applying the trained classifier $D$ individually to each instance in the set $X$, and a greedy approach where objects and classes are paired and eliminated from the set. Our experimental study validates the proposed approach for various choices of base classifier model $D$.

We demonstrated that the set classifier is better than the individual classifier for the restricted set classification problem through a two-part experiment. In the first part, we formulated the problem on a chess dataset where the all pieces on a chessboard had to be recognised from a bird-view snapshot. The second experiment was carried out using the University of Santiago de Compostela collection of 96 data. We constructed restricted set classification problems and applied the proposed solution. The experiments 
favoured ensemble classifiers as the base classifier $D$, and subsequently the Hungarian set classifier $D_{\text {set }}^{h}$ on the expanded probability matrix.

The scalability of the proposed framework is affected only by the complexity of the Hungarian algorithm. Training of the base classifier $D$ is the same with or without the restricted set classification framework. The complexity of the Hungarian algorithm for our case is $O\left(k^{3}\right)$, where $k$ is the maximum number of objects in the set. The Hungarian algorithm may be impractical for very large $k$ or when rapid classification is needed, for example in tracking. In such cases, the greedy set classifier can be used, sacrificing some accuracy $A_{P}$ and $A_{T}$.

An interesting question which we intend to address in the future is that about possible dependencies in the set of objects $X$. The instances in $X$ may not be independently drawn from their respective classes. For example, the snapshot of the chessboard has a certain illumination. When classifying the pieces in the individual squares cropped from the snapshot, we assume that they are drawn independently from their respective classes. In other words, the fact that these sub-images have similar illumination is not currently accounted for in the theoretical model we propose here.

\section{Acknowledgements}

This work was partially supported by the Spanish Ministry of Economy and Competitiveness through project TIN 2015-67534-P.

\section{Appendix}

Proof of Proposition 1

Proposition 1. For 2-class problems,

$$
A_{P}\left(D_{\text {set }}^{g}\right)>A_{P}\left(D_{\text {set }}^{i}\right)
$$

Proof. For $D_{\text {set }}^{i}$,

$$
\begin{aligned}
A_{P}\left(D_{\mathrm{set}}^{i}\right) & =\operatorname{Pr}\left(P_{1}>0.5\right) \operatorname{Pr}\left(P_{2}>0.5\right) \times \frac{2}{2} \\
& +\operatorname{Pr}\left(P_{1}>0.5\right) \operatorname{Pr}\left(P_{2} \leq 0.5\right) \times \frac{1}{2} \\
& +\operatorname{Pr}\left(P_{1} \leq 0.5\right) \operatorname{Pr}\left(P_{2}>0.5\right) \times \frac{1}{2} \\
& =\frac{1}{2}\left(\operatorname{Pr}\left(P_{1}>0.5\right)+\operatorname{Pr}\left(P_{2}>0.5\right)\right) \\
& =p,
\end{aligned}
$$


where $p$ is the accuracy of $D$. For $D_{\text {set }}^{g}$,

$$
\begin{aligned}
& A_{P}\left(D_{\text {set }}^{g}\right)=\operatorname{Pr}\left(P_{1}>0.5\right) \operatorname{Pr}\left(P_{2}>0.5\right) \times \frac{2}{2} \\
& +\operatorname{Pr}\left(P_{1} \leq 0.5\right) \operatorname{Pr}\left(P_{2}>0.5\right) \operatorname{Pr}\left(P_{2}>1-P_{1}\right) \times \frac{2}{2} \\
& +\operatorname{Pr}\left(P_{1}>0.5\right) \operatorname{Pr}\left(P_{2} \leq 0.5\right) \operatorname{Pr}\left(P_{1}>1-P_{2}\right) \times \frac{2}{2} .
\end{aligned}
$$

Denote by $S_{1}=\operatorname{Pr}\left(P_{1}>0.5\right)$ the sensitivity of $D$ assuming that class $\omega_{1}$ is the positive class, and by $S_{2}=\operatorname{Pr}\left(P_{2}>0.5\right)$ the sensitivity of $D$ assuming that class $\omega_{2}$ is the positive class. Then

$$
\begin{aligned}
A_{P}\left(D_{\text {set }}^{g}\right) & =S_{1} S_{2}+\left(1-S_{1}\right) S_{2} A U C+S_{1}\left(1-S_{2}\right) A U C \\
& =S_{1} S_{2}+\left(S_{1}+S_{2}-2 S_{1} S_{2}\right) A U C .
\end{aligned}
$$

For the Bayes classifier $D$, whereby the labelling is done by the largest posterior probability, $A U C>0.5$. Therefore,

$$
A_{P}\left(D_{\text {set }}^{g}\right)>S_{1} S_{2}+\left(S_{1}+S_{2}-2 S_{1} S_{2}\right) \times 0.5
$$

390

$$
=\frac{1}{2}\left(S_{1}+S_{2}\right)=p=A_{P}\left(D_{\text {set }}^{i}\right) .
$$


Results for the USC data for the decision tree classifier

Table 4: Averaged partial accuracy $A_{P}$ and averaged total accuracy $A_{T}$ in $\%$ for the Baseline (B), Greedy (G) and the Hungarian $(\mathrm{H})$ set classifiers for the decision tree classifier. Symbol • after the value in column $\mathrm{H}$ indicates that $\mathrm{H}$ is significantly different from $\mathrm{G}$ at $p<0.01$. The highest accuracy for each dataset is indicated in boldface.

\begin{tabular}{|c|c|c|c|c|c|c|}
\hline \multirow[b]{2}{*}{ Classifier } & \multicolumn{3}{|c|}{$A_{P}$} & \multicolumn{3}{|c|}{$A_{T}$} \\
\hline & B & G & $\mathrm{H}$ & B & G & $\mathrm{H}$ \\
\hline abalone & 61.01 & 63.06 & $63.23-$ & 6.50 & 7.60 & $7.60-$ \\
\hline acute-inflammation & 100.00 & 100.00 & $100.00-$ & 100.00 & 100.00 & $100.00-$ \\
\hline acute-nephritis & 100.00 & 100.00 & $100.00-$ & 100.00 & 100.00 & $100.00-$ \\
\hline adult & 76.34 & 79.90 & $79.95-$ & 28.10 & 34.80 & $35.00-$ \\
\hline annealing & 90.64 & 91.60 & $91.99-$ & 52.10 & 56.10 & $57.50-$ \\
\hline arrhythmia & 82.84 & 84.79 & $85.41-$ & 41.70 & 46.40 & $47.80-$ \\
\hline balance-scale & 85.11 & 85.52 & $85.52-$ & 43.50 & 45.90 & $46.10-$ \\
\hline bank & 64.79 & 71.92 & $71.94-$ & 18.60 & 23.90 & $24.70-$ \\
\hline blood & 60.04 & 68.82 & $69.92-$ & 15.90 & 23.10 & $25.60-$ \\
\hline breast-cancer-wisc-diag & 92.85 & 92.61 & $92.95-$ & 64.70 & 66.50 & $67.40-$ \\
\hline breast-cancer-wisc & 94.26 & 94.34 & $94.32-$ & 70.50 & 72.50 & $72.40-$ \\
\hline breast-cancer & 58.66 & 65.34 & $66.58-$ & 11.90 & 17.50 & $19.30-$ \\
\hline car & 92.04 & 92.58 & $92.78-$ & 46.70 & 50.20 & $51.30-$ \\
\hline cardiotocography-10clases & 74.96 & 74.96 & $74.77-$ & 0.50 & 0.60 & $0.60-$ \\
\hline cardiotocography-3clases & 85.53 & 86.14 & $86.09-$ & 34.10 & 37.60 & $37.40-$ \\
\hline chess-krvk & 73.36 & 74.25 & $74.48 \bullet$ & 0.00 & 0.10 & $0.10-$ \\
\hline chess-krvkp & 98.99 & 99.04 & $99.09-$ & 94.70 & 95.30 & $95.70-$ \\
\hline congressional-voting & 48.95 & 62.61 & $67.07 \bullet$ & 11.80 & 21.10 & $26.10 \bullet$ \\
\hline conn-bench-sonar-mines-ro & 69.99 & 72.29 & $72.30-$ & 19.70 & 23.10 & $23.50-$ \\
\hline conn-bench-vowel-deterdin & 81.38 & 81.22 & $81.60 \bullet$ & 1.70 & 1.80 & $1.90-$ \\
\hline connect- 4 & 74.75 & 77.78 & $77.79-$ & 24.70 & 29.00 & $29.10-$ \\
\hline contrac & 48.18 & 50.17 & $50.79 \bullet$ & 2.80 & 2.90 & $2.90-$ \\
\hline credit-approval & 84.22 & 84.32 & $84.32-$ & 37.90 & 40.80 & $40.80-$ \\
\hline cylinder-bands & 68.71 & 70.59 & $70.67-$ & 20.20 & 23.00 & $22.90-$ \\
\hline dermatology & 96.86 & 96.81 & $96.93-$ & 73.40 & 74.70 & $74.90-$ \\
\hline ecoli & 91.02 & 91.01 & $92.10 \bullet$ & 51.70 & 53.20 & $56.30 \bullet$ \\
\hline energy-y1 & 93.55 & 93.90 & $94.11-$ & 59.20 & 63.40 & $64.80-$ \\
\hline energy-y2 & 88.81 & 89.57 & $89.83-$ & 39.90 & 44.20 & $45.00-$ \\
\hline glass & 73.44 & 75.24 & $75.70-$ & 23.10 & 26.80 & $27.90-$ \\
\hline haberman-survival & 60.01 & 66.97 & $68.40-$ & 14.00 & 19.30 & $21.40 \bullet$ \\
\hline hayes-roth & 61.75 & 65.71 & $66.39-$ & 15.50 & 19.50 & $20.30-$ \\
\hline heart-cleveland & 68.21 & 73.01 & $73.63 \bullet$ & 19.70 & 24.40 & $25.40 \bullet$ \\
\hline heart-hungarian & 76.13 & 78.80 & $79.00-$ & 26.80 & 31.20 & $31.90-$ \\
\hline heart-va & 54.55 & 58.22 & $58.32-$ & 9.40 & 11.10 & $11.40-$ \\
\hline hill-valley & 50.59 & 65.25 & $70.34 \bullet$ & 14.90 & 26.10 & $31.70 \bullet$ \\
\hline horse-colic & 81.21 & 82.36 & $82.58-$ & 34.40 & 38.00 & $38.50-$ \\
\hline ilpd-indian-liver & 52.41 & 63.11 & $65.28-$ & 11.60 & 19.70 & $22.60-$ \\
\hline image-segmentation & 93.57 & 93.40 & $93.39-$ & 29.90 & 32.00 & $32.20-$ \\
\hline ionosphere & 83.95 & 83.71 & $83.78-$ & 40.70 & 42.40 & $42.40-$ \\
\hline iris & 92.99 & 92.73 & $92.97-$ & 60.40 & 62.20 & $63.20-$ \\
\hline led-display & 70.21 & 70.25 & $70.20-$ & 0.10 & 0.10 & $0.10-$ \\
\hline
\end{tabular}


Table 4 - continued from previous page

\begin{tabular}{|c|c|c|c|c|c|c|}
\hline \multirow[b]{2}{*}{ Classifier } & \multicolumn{3}{|c|}{$A_{P}$} & \multicolumn{3}{|c|}{$A_{T}$} \\
\hline & B & G & $\mathrm{H}$ & $\mathrm{B}$ & G & $\mathrm{H}$ \\
\hline letter & 84.33 & 84.04 & $84.03-$ & 0.00 & 0.00 & $0.00-$ \\
\hline low-res-spect & 80.94 & 81.65 & $81.76-$ & 23.60 & 26.40 & $27.20-$ \\
\hline lymphography & 75.82 & 78.33 & $78.61-$ & 27.30 & 32.00 & $33.10-$ \\
\hline magic & 82.35 & 83.81 & $83.78-$ & 38.60 & 42.80 & $42.80-$ \\
\hline mammographic & 83.16 & 84.03 & $84.27-$ & 37.10 & 41.50 & $42.20 \bullet$ \\
\hline miniboone & 87.53 & 87.72 & $87.72-$ & 48.60 & 50.80 & $50.80-$ \\
\hline molec-biol-promoter & 74.64 & 76.13 & $76.48-$ & 28.10 & 31.00 & $31.90-$ \\
\hline molec-biol-splice & 89.73 & 89.55 & $89.45-$ & 45.30 & 47.40 & $47.40-$ \\
\hline monks-1 & 83.45 & 86.07 & $87.22-$ & 47.00 & 52.70 & $54.80-$ \\
\hline monks-2 & 58.44 & 67.65 & $69.38-$ & 13.60 & 22.70 & $25.10-$ \\
\hline monks-3 & 96.97 & 97.26 & $97.32-$ & 83.30 & 85.60 & $85.80-$ \\
\hline mushroom & 100.00 & 100.00 & $100.00-$ & 100.00 & 100.00 & $100.00-$ \\
\hline musk-1 & 76.64 & 77.30 & $77.79-$ & 27.80 & 31.20 & $32.30-$ \\
\hline musk-2 & 90.16 & 90.58 & $90.64-$ & 56.70 & 60.20 & $60.30-$ \\
\hline nursery & 98.36 & 98.54 & $98.57-$ & 83.90 & 86.10 & $86.30-$ \\
\hline oocytes-merluccius-nucleu & 64.59 & 68.07 & $68.11-$ & 16.20 & 19.10 & $19.60-$ \\
\hline oocytes-merluccius-states & 83.63 & 83.30 & $83.31-$ & 28.90 & 30.30 & $30.60-$ \\
\hline oocytes-trisopterus-nucle & 69.67 & 71.35 & $71.48-$ & 18.30 & 20.10 & $20.30-$ \\
\hline oocytes-trisopterus-state & 85.77 & 86.15 & $86.19-$ & 43.90 & 47.20 & $47.60-$ \\
\hline optical & 88.81 & 88.44 & $88.47-$ & 5.70 & 6.20 & $6.30-$ \\
\hline ozone & 57.82 & 66.25 & $66.89-$ & 16.00 & 21.60 & $22.70-$ \\
\hline page-blocks & 84.40 & 85.49 & $86.00 \bullet$ & 26.20 & 29.30 & $30.10-$ \\
\hline pendigits & 95.13 & 94.83 & $94.84-$ & 27.90 & 29.10 & $29.00-$ \\
\hline pima & 69.17 & 72.39 & $72.77-$ & 20.20 & 24.50 & $25.10-$ \\
\hline planning & 51.08 & 65.87 & $71.35 \bullet$ & 12.80 & 25.40 & $30.80 \bullet$ \\
\hline ringnorm & 89.81 & 90.05 & $90.18-$ & 53.90 & 57.30 & $57.30-$ \\
\hline seeds & 90.40 & 90.55 & $90.49-$ & 49.10 & 51.80 & $52.20-$ \\
\hline semeion & 72.11 & 71.62 & $71.73-$ & 0.10 & 0.10 & $0.10-$ \\
\hline soybean & 86.94 & 87.47 & $87.94 \bullet$ & 28.10 & 30.90 & $32.50 \bullet$ \\
\hline spambase & 90.87 & 91.19 & $91.14-$ & 58.10 & 61.70 & $61.80-$ \\
\hline spect & 65.84 & 68.54 & $69.22-$ & 15.50 & 18.90 & $19.60-$ \\
\hline spectf & 70.13 & 71.11 & $70.51-$ & 21.70 & 23.30 & $23.70-$ \\
\hline statlog-australian-credit & 54.57 & 57.59 & $58.14-$ & 10.30 & 11.80 & $12.30-$ \\
\hline statlog-german-credit & 62.43 & 66.31 & $66.25-$ & 13.80 & 16.60 & $16.60-$ \\
\hline statlog-heart & 74.53 & 76.02 & $75.77-$ & 24.10 & 27.20 & $27.30-$ \\
\hline statlog-image & 95.05 & 94.96 & $95.02-$ & 40.20 & 41.80 & $41.90-$ \\
\hline statlog-landsat & 82.25 & 82.04 & $82.19-$ & 8.60 & 9.10 & $9.50-$ \\
\hline statlog-shuttle & 98.15 & 98.25 & $98.18-$ & 79.30 & 81.00 & $80.60-$ \\
\hline statlog-vehicle & 70.48 & 71.81 & $72.00-$ & 7.30 & 8.40 & $8.60-$ \\
\hline steel-plates & 71.98 & 72.57 & $72.72-$ & 0.80 & 1.10 & $1.10-$ \\
\hline synthetic-control & 87.35 & 86.84 & $87.24-$ & 14.20 & 15.00 & $15.60-$ \\
\hline teaching & 58.33 & 62.62 & $62.83-$ & 9.60 & 12.70 & $13.30-$ \\
\hline thyroid & 97.59 & 97.57 & $97.55-$ & 82.80 & 84.20 & $84.00-$ \\
\hline tic-tac-toe & 91.11 & 91.69 & $91.64-$ & 61.00 & 64.60 & $64.50-$ \\
\hline titanic & 68.37 & 75.28 & $76.43 \bullet$ & 22.40 & 32.50 & $34.60 \bullet$ \\
\hline twonorm & 84.40 & 84.54 & $84.62-$ & 40.30 & 42.90 & $43.10-$ \\
\hline vertebral-column-2clases & 76.37 & 78.38 & $78.63-$ & 29.30 & 33.30 & $33.70-$ \\
\hline vertebral-column-3clases & 74.11 & 75.90 & $75.77-$ & 16.70 & 19.50 & $19.60-$ \\
\hline wall-following & 99.13 & 99.13 & $99.18 \bullet$ & 90.00 & 90.50 & $91.00-$ \\
\hline
\end{tabular}




\begin{tabular}{l|rrc|rrr}
\multicolumn{7}{c}{ Table 4 continued from previous page } \\
& \multicolumn{7}{c}{$A_{P}$} & & $A_{T}$ & \\
Classifier & $\mathrm{B}$ & $\mathrm{G}$ & $\mathrm{H}$ & $\mathrm{B}$ & $\mathrm{G}$ & $\mathrm{H}$ \\
\hline waveform-noise & 74.58 & 74.70 & $\mathbf{7 4 . 9 7}-$ & 14.20 & 15.30 & $\mathbf{1 5 . 7 0}-$ \\
waveform & 76.16 & 76.40 & $\mathbf{7 6 . 6 8}-$ & 17.10 & 18.20 & $\mathbf{1 8 . 4 0}-$ \\
wine-quality-red & 42.66 & 46.18 & $\mathbf{4 6 . 5 4}-$ & 1.00 & $\mathbf{1 . 3 0}$ & $\mathbf{1 . 3 0}-$ \\
wine-quality-white & 42.51 & 44.73 & $\mathbf{4 6 . 1 6} \bullet$ & 0.20 & 0.20 & $\mathbf{0 . 3 0}-$ \\
wine & 94.15 & 94.03 & $\mathbf{9 4 . 4 8}-$ & 71.80 & 73.60 & $\mathbf{7 5 . 1 0} \bullet$ \\
yeast & 57.58 & 58.41 & $\mathbf{5 8 . 6 2}-$ & $\mathbf{1 . 2 0}$ & $\mathbf{1 . 2 0}$ & $\mathbf{1 . 2 0}-$
\end{tabular}

\section{References}

[1] L. I. Kuncheva, Full-class set classification using the Hungarian algorithm, International Journal of Machine Learning and Cybernetics 1 (1) (2010) 53-61. doi:D0I10.1007/s13042-010-0002-z

[2] L. I. Kuncheva, A. S. Jackson, Who is missing? A new pattern recognition puzzle, in: Intenrational Conference on Statistical, Structural and Syntactic Pattern Recognition (S+SSPR), Vol. LNCS 8621, Springer, Joensuu, Funland, 2014, pp. 243-252.

[3] R. O. Duda, P. E. Hart, D. G. Stork, Pattern Classification, 2nd Edition, John Wiley \& Sons, NY, 2001.

[4] R. E. Slot, On the profit of taking into account the known number of objects per class in classification methods IT-25 (1979) 484-488.

[5] T. G. Dietterich, R. H. Lathrop, T. Lozano-Perez, Solving the multiple-instance problem with axis-parallel rectangles, Artificial Intelligence 89 (1997) 31-71.

[6] J. Wang, J.-D. Zucker, Solving the multiple-instance problem: A lazy learning approach, in: In Proceedings 17th International Conference on Machine Learning, 2000, pp. 1119-1125.

[7] O. L. Mangasarian, E. W. Wild, Multiple instance classification via successive linear programming, Journal of Optimization Theory and Applications 137 (2008) 555-568.

[8] Z. Fu, A. Robles-Kelly, J. Zhou, MILIS: multiple instance learning with instance selection, IEEE Transactions on Pattern Analysis and Machine Intelligence 33 (5) (2011) 958-977.

[9] X. Xu, Statistical learning in multiple instance problems, Master's thesis, University of Waikato, Department of Computer Science, New Zealand (2003).

[10] X. Ning, G. Karypis, The set classification problem and solution methods, in: In Proceedings of SIAM Data Mining, 2009, pp. 847-858.

[11] M. Pelillo, M. Refice, Learning compatibility coefficients for relaxation labeling processes, Pattern Analysis and Machine Intelligence, IEEE Transactions on 16 (9) (1994) 933-945.

[12] A. Rosenfeld, R. A. Hummel, S. Zucker, Scene labeling by relaxation operations, IEEE Transactions on Systems, Man and Cybernetics SMC-6 (6) (1976) 420-433.

[13] L. K. McDowell, K. M. Gupta, D. W. Aha, Cautious inference in collective classification, in: In Processdings of AAAI, 2007, pp. 596-601.

[14] P. Sen, G. M. Namata, Bilgic, L. Getoor, B. Gallagher, T. Eliassi-Rad, Collective classification in network data, AI Magazine 29 (2008) 93106.

[15] Y. Amit, A. Trouvé, POP: patchwork of parts models for object recognition, International Journal of Computer Vision 75 (2007) 267-282.

[16] R. Kaucic, A. G. A. Perera, G. B. J., Kaufhold, A. Hoogs, A unified framework for tracking through occlusions and across sensor gaps, in: IEEE Computer Society Conference on Computer Vision and Pattern Recognition, CVPR, Vol. 1, 2005, pp. 1063-1069. 
[17] I. Haritaoglu, D. Harwood, L. S. Davis, W 4: Real-time surveillance of people and their activities, Pattern Analysis and Machine Intelligence, IEEE Transactions on 22 (8) (2000) 809-830.

[18] A. Mohan, C. Papageorgiou, T. Poggio, Example-based object detection in images by components, Pattern Analysis and Machine Intelligence, IEEE Transactions on 23 (4) (2001) 349-361.

[19] P. F. Felzenszwalb, D. P. Huttenlocher, Pictorial structures for object recognition, International Journal of Computer Vision 61 (1) (2005) 55-79.

[20] B. Heisele, T. Serre, M. Pontil, T. Poggio, Component-based face detection, in: Computer Vision and Pattern Recognition, 2001. CVPR 2001. Proceedings of the 2001 IEEE Computer Society Conference on, Vol. 1, IEEE, 2001 , pp. I-657.

[21] T. Zhao, R. Nevatia, Tracking multiple humans in complex situations, Pattern Analysis and Machine Intelligence, IEEE Transactions on 26 (9) (2004) 1208-1221.

[22] X. Zhou, Y. Li, B. He, Game-theoretical occlusion handling for multi-target visual tracking, Pattern Recognition 46 (10) (2013) 2670-2684.

[23] C. Rasmussen, G. D. Hager, Probabilistic data association methods for tracking complex visual objects, Pattern Analysis and Machine Intelligence, IEEE Transactions on 23 (6) (2001) 560-576.

[24] Y. Bar-Shalom, F. Daum, J. Huang, The probabilistic data association filter, Control Systems, IEEE 29 (6) (2009) 82-100.

[25] K. Okuma, A. Taleghani, N. De Freitas, J. J. Little, D. G. Lowe, A boosted particle filter: Multitarget detection and tracking, in: Computer Vision-ECCV 2004, Springer, 2004, pp. 28-39.

[26] A. Dearden, Y. Demiris, O. Grau, Tracking football player movement from a single moving camera using particle filters, in: Proceedings of CVMP-2006, IET Press, 2006, pp. 29-37.

[27] P. Konstantinova, A. Udvarev, T. Semerdjiev, A study of a target tracking algorithm using global nearest neighbor approach, in: Proceedings of the International Conference on Computer Systems and Technologies (CompSysTech03), 2003.

[28] R. Ardekani, A. Biyani, J. E. Dalton, J. B. Saltz, M. N. Arbeitman, J. Tower, S. Nuzhdin, S. Tavaré, Three-dimensional tracking and behaviour monitoring of multiple fruit flies, Journal of The Royal Society Interface (2012) rsif20120547.

[29] J. N. Jover, M. Alcañiz-Raya, V. Gómez, S. Balasch, J. Moreno, V. G. Colomer, A. Torres, An automatic colour-based computer vision algorithm for tracking the position of piglets, Spanish Journal of Agricultural Research 7 (3) (2009) $535-549$.

[30] M. Kashiha, C. Bahr, S. Ott, C. P. Moons, T. A. Niewold, F. O. Ödberg, D. Berckmans, Automatic identification of marked pigs in a pen using image pattern recognition, Computers and Electronics in Agriculture 93 (2013) 111-120.

[31] Y. Kamisugi, N. Furuya, K. Iijima, K. Fukui, Computer-aided automatic identification of rice chromosomes by image parameters 1 (3) (1993) 189-196.

[32] D. Ming, J. Tian, Automatic pattern extraction and classification for chromosome images, Journal of Infrared Milli Terahz Waves 31 (2010) 866-877.

[33] X. Wang, S. Li, H. Liu, M. Wood, W. R. Chen, B. Zheng, Automated identification of analyzable metaphase chromosomes depicted on microscopic digital images, J Biomed Inform 41 (2) (2008) 264-271.

[34] H. W. Kuhn, The Hungarian Method for the assignment problem, Naval Research Logistic Quarterly 2 (1955) 83-97.

[35] F. Bourgeois, J.-C. Lassalle, An extension of the Munkres algorithm for the assignment problem to rectangular matrices, Communications ACM 14 (12) (1971) 802-804.

[36] C.-C. Chang, C.-J. Lin, LIBSVM: A library for support vector machines, ACM Transactions on Intelligent Systems and Technology 2 (2011) 27:1-27:27, software available at http://www.csie.ntu.edu.tw/ cjlin/libsvm

[37] M. Fernández-Delgado, E. Cernadas, S. Barro, D. Amorim, Do we need hundreds of classifiers to solve real world classification problems?, Journal of Machine Learning Research 15 (2014) 3133-3181.

[38] K. Bache, M. Lichman, UCI machine learning repository (2013). 
URL http://archive.ics.uci.edu/ml

[39] T. Fawcett, ROC graphs: Notes and practical considerations for researchers, Tech. Rep. HPL-2003-4, HP Labs, Palo Alto, http://www.hpl.hp.com/techreports/2003/HPL-2003-4.pdf (2003).

\section{Vitae}

Ludmila I. Kuncheva is a Professor of Computer Science at Bangor University, UK. Her interests include pattern recognition, and specifically classifier ensembles. She has published two monographs and over 150 research papers. Lucy has won two Best Paper Awards (2006 IEEE TFS and 2003 IEEE TSMC.) She is a Fellow of IAPR.

Juan J. Rodríguez is an Associate Professor of Computer Science at University of Burgos, Spain. His interests include data science, pattern recognition and specifically classifier ensembles.

Aaron S. Jackson is currently working toward his $\mathrm{PhD}$ degree in Computer Science at Nottingham University, UK. His interests include pattern recognition, deep learning and computer vision. 